\title{
PUMP IT UP! USE OF REBOA IN BLUNT ABDOMINAL AORTIC INJURY (BAAI): A CASE REPORT
}

\section{Chiarini ${ }^{1}$, G. Mignani' ${ }^{2}$, E. Matteo ${ }^{1}$, C. Lupi ${ }^{2}$, E. Ferri' ${ }^{2}$, C. Coniglio ${ }^{2}$, G. Gordini ${ }^{2}$}

${ }^{1}$ Anesthesia and Intensive Care, University of Bologna ${ }^{2}$ Anesthesia, Intensive Care and EMS Department, Maggiore Hospital, Bologna, Italy

\section{Purpose}

To evaluate the feasibility of using Resuscitative Endovascular Balloon Occlusion of the Aorta (REBOA) in BAAI. \begin{abstract}
(1)
Results

Patient's Injury Severity Score was 75. Other main injuries were subarachnoid hemorrhage, massive hemothorax, pelvic and vertebral fractures. Total inflation time of REBOA was 30 minutes in zone $3,45 \mathrm{~min}$, intermittent, in zone 2. Early Continuous renal replacement therapy was started to prevent crush syndrome consequences. The patient suffered from transient intestinal impairment and acute limb compartment syndrome as major complications, related to ischemia-reperfusion injury.
\end{abstract}

\section{Conclusions}

Indication for the use of REBOA in BAAl is still lacking, due to potential risk of extension of the aortic lesion. Nevertheless, the technique could represent a bridge to get to definitive treatment in life-threatening situations. We hypothesize that when trauma dynamic poses a strong suspicion of BAAl it could be useful to place REBOA with fluoroscopic or CT-scan guidance in order to exclude the lesion while minimizing splancnic ischaemic injury. Despite the contraindication to place REBOA in zone 2, this approach seems to be feasible and relatively safe.
Trauma scores

RTS $=5,148$

SBP $60 \mathrm{mmHg}$

GCS 11 (E3 V2 M6)

ISS $=75$

hAIS 5

tAIS 5

aAIS 5

eAIS 4
REBOA in

zone 3

(between

renal arteries

and aortic

bifurcation) 